\title{
A influência simultânea do teor de alumina, da porosidade total e da temperatura na condutividade térmica de refratários sílico-aluminosos e aluminosos
}

\section{(The simultaneous influence of alumina content, total porosity and temperature on the thermal conductivity of fireclay and alumina refractories)}

\author{
M. M. Akiyoshi, M. G. da Silva, A. P. da Silva, V. C. Pandolfelli \\ Via Washington Luiz, km 235, C.P. 676, 13565-905, S. Carlos, SP. \\ Universidade Federal de São Carlos \\ pmmak@iris.ufscar.brou vicpando@power.ufscar.br
}

\begin{abstract}
Resumo
A condutividade térmica de materiais refratários sílicoaluminosos e aluminosos com $35,81 \%$-p $\leq \mathrm{Al}_{2} \mathrm{O}_{3} \leq 93,48 \%$ p e porosidade total entre $15 \%$ e $81 \%$ foi determinada através da técnica de fio quente paralelo. Além de avaliar as correlações entre a condutividade térmica, o teor de alumina $\left(\mathrm{Al}_{2} \mathrm{O}_{3}\right)$ e a porosidade (PA e PT), neste trabalho, foi introduzida a razão R (teor de alumina / porosidade total) que permitiu correlacionar a condutividade térmica simultaneamente com a composição química e a microestrutura dos refratários. O método dos mínimos quadrados foi utilizado para generalizar a correlação entre k e R para uma faixa mais ampla de temperaturas. A boa qualidade do ajuste alcançado pelo modelo empírico obtido $\left(\mathrm{r}^{2}>0,93\right)$ permite concluir que a utilização da razão $R$ pode ser mais significa para a condutividade térmica de cerâmicas refratárias do que a utilização do teor de alumina ou quantidade de coríndon isoladamente. Estas relações são fundamentais no projeto de fornos e equipamentos que empreguem cerâmicas refratárias além de serem dados de entrada adequados para suprir programas de simulação computacional que visem à previsão do comportamento termomecânico destes materiais.
\end{abstract}

Palavras-chave: materiais refratários, condutividade térmica.

\section{INTRODUÇÃO}

A crescente competitividade no setor siderúrgico tem impulsionado não só a melhoria dos revestimentos refratários existentes, como também o desenvolvimento de materiais que sejam adequados às mais diversas aplicações. Como a maioria dos materiais refratários é empregada em condições severas de temperatura, tornou-se fundamental o conhecimento de suas propriedades térmicas, dentre as quais destaca-se a condutividade térmica $(\mathrm{k})$.

Vários trabalhos na literatura [1-4] correlacionaram a

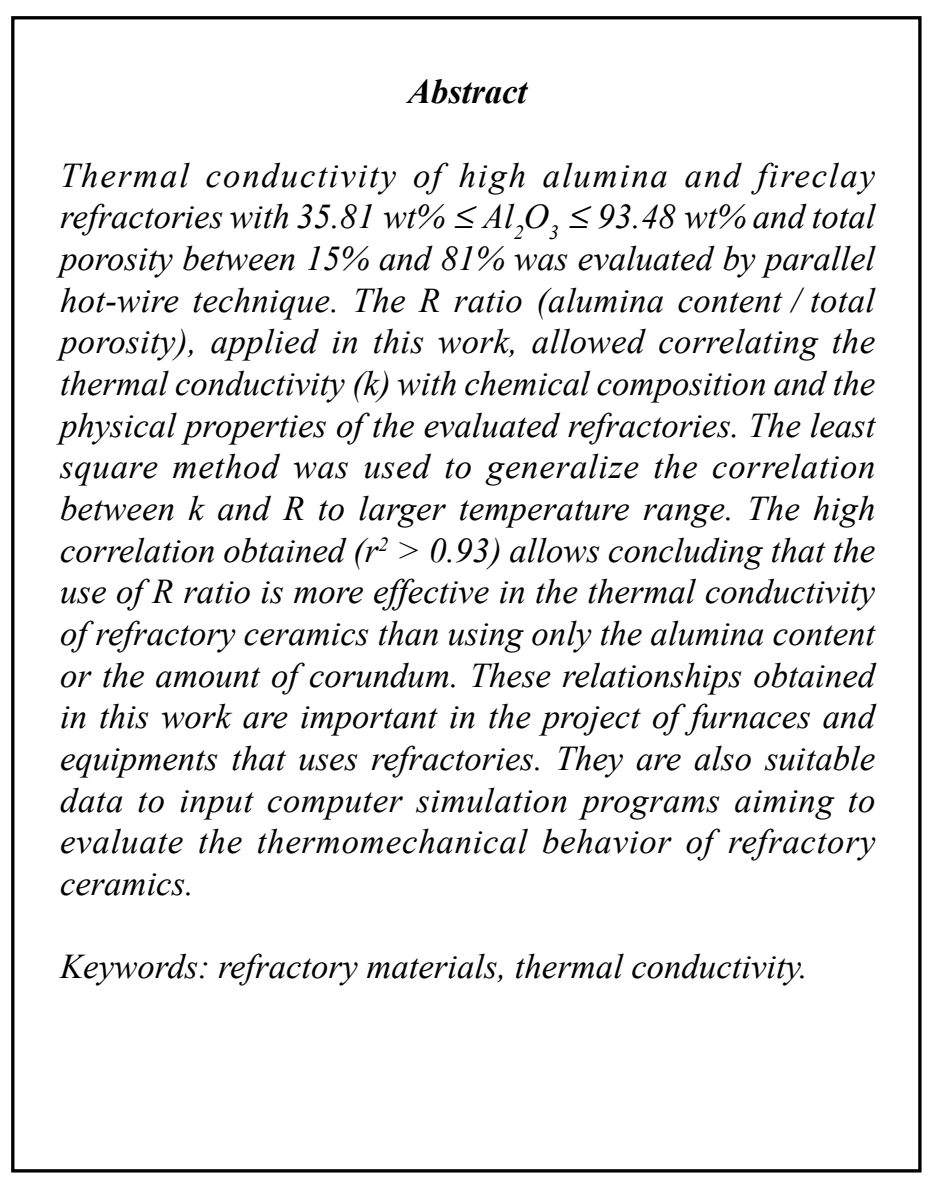

condutividade térmica com a massa específica geométrica $\left(\rho_{\mathrm{g}}\right)$ ou volumétrica $\left(\rho_{\mathrm{v}}\right)$, encontrando relações exponenciais como as expressas pela equação (A) na qual $\mathrm{p}_{1}$ e $\mathrm{p}_{2}$ são parâmetros a serem determinados.

$$
\mathrm{k}=\mathrm{p}_{1}+\exp \left(\mathrm{p}_{2} \cdot \rho_{\mathrm{v}}\right)
$$

Diferentemente, Akiyoshi e colaboradores [5] apresentaram uma função que correlacionava simultaneamente a condutividade térmica, a massa específica volumétrica e a temperatura, como apresentado pela equação (B). 
$\mathrm{k}=\exp \left[\begin{array}{c}-2,8915+0,1543 \cdot 10^{-2} \cdot \mathrm{T}-0,4908 \cdot 10^{-6} \cdot \mathrm{T}^{2}+ \\ +\left(1,5549-0,9277 \cdot 10^{-3} \cdot \mathrm{T}+0,4095 \cdot 10^{-6} \cdot \mathrm{T}^{2}\right) \rho_{\mathrm{v}}\end{array}\right](\mathrm{B})$
$\quad\left(25^{\circ} \mathrm{C} \leq \mathrm{T} \leq 1200^{\circ} \mathrm{C} \quad\right.$ e $\left.\quad 0,55 \mathrm{~g} / \mathrm{cm}^{3} \leq \rho_{\mathrm{v}} \leq 3,14 \mathrm{~g} / \mathrm{cm}^{3}\right)$

Na qual T é a temperatura $\left({ }^{\circ} \mathrm{C}\right)$ e $\rho_{\mathrm{v}}$ é a massa específica volumétrica $\left(\mathrm{g} / \mathrm{cm}^{3}\right)$ e a condutividade térmica k expressa em $(\mathrm{W} / \mathrm{m} \cdot \mathrm{K})$.

Outros trabalhos buscaram correlações entre a condutividade térmica $(\mathrm{k})$ e o teor de alumina $\left(\mathrm{Al}_{2} \mathrm{O}_{3}\right)$ [6] ou entre a condutividade térmica e a quantidade de agregados grosseiros [7]. Chien e colaboradores [6] estudaram refratários com teor de alumina no intervalo $44,20 \%$-p $\leq \mathrm{Al}_{2} \mathrm{O}_{3} \leq 92,77 \%$-p e porosidade total entre $9,5 \%$ e $23,56 \%$. Estes autores concluíram que a condutividade térmica não aumentava obrigatoriamente com o teor de $\mathrm{Al}_{2} \mathrm{O}_{3}$, mas sim, com a quantidade de alumina presente sob a forma de coríndon. Essa conclusão também foi obtida por Chan e Ko [7] que utilizaram refratários com $90 \%$ - $\mathrm{p} \leq \mathrm{Al}_{2} \mathrm{O}_{3} \leq 96 \%$-p e porosidade aparente entre $19,38 \%$ e $25,95 \%$. Chan e Ko verificaram que a quantidade de coríndon e a presença de agregados de alumina de maior pureza eram mais importantes que a quantidade de agregados grosseiros. Todavia, nestes trabalhos não foi considerada a influência da porosidade sobre a condutividade térmica.

Diferentemente, neste trabalho, além de se estudar as correlações entre a condutividade térmica, o teor de alumina e a porosidade, individualmente, buscou-se uma correlação que contemplasse simultaneamente os efeitos do teor de alumina e da porosidade total (PT) sobre a condutividade térmica. Para isso, foi utilizada a razão. $\mathrm{R}=\frac{\text { Teor de alumina }}{\text { Porosidade total }}$. Em seguida, determinou-se uma expressão para a condutividade térmica em função da razão $\mathrm{R}$ e da temperatura.

Tais relações são fundamentais no projeto de fornos e equipamentos que utilizem materiais refratários, além de gerarem dados de entrada adequados para suprir programas de simulação computacional que visam à previsão de seu comportamento termomecânico.

\section{MATERIAIS E MÉTODOS}

Materiais utilizados. Todos os materiais deste trabalho foram tratados termicamente em temperaturas superiores àquelas utilizadas na avaliação da condutividade térmica. A Tabela I apresenta o teor de alumina (\%-p), a porosidade total (\%) e a razão $\mathrm{R}(-)$ dos refratários utilizados. As análises químicas completas e as características físicas dos refratários deste trabalho, bem como a metodologia utilizada em sua avaliação podem ser encontradas em Akiyoshi e colaboradores [5].

Avaliação da condutividade térmica. A condutividade térmica dos materiais refratários foi avaliada através da técnica de fio quente paralelo. Essa técnica tem natureza transiente e permite avaliar diretamente (sem a necessidade de padrões calibrados) condutividades térmicas entre $0,10 \mathrm{~W} / \mathrm{m} \cdot \mathrm{K}$ e $25 \mathrm{~W} / \mathrm{m} \cdot \mathrm{K}$. Pelo fato de ser transiente, utiliza um pequeno gradiente térmico, permitindo a avaliação da condutividade térmica tanto em baixas temperaturas (como a ambiente) como em temperaturas elevadas $\left(1250^{\circ} \mathrm{C}\right)$. Emprega corpos-de-prova de dimensões realistas $(230 \times 114 \times 64) \mathrm{mm}^{3}$, o que é muito importante para materiais de microestrutura grosseira, como os refratários, possibilitando a obtenção de valores mais represen- tativos. O modelo teórico no qual se baseia a técnica de fio quente paralelo bem como a descrição dos equipamentos e dos procedimentos utilizados para a caracterização dos materiais pode ser encontrada em trabalho anterior [5]. Neste trabalho foram empregadas as seguintes temperaturas de ensaio: 25, 200, 400, 600, 800, 1000 e $1200^{\circ} \mathrm{C}$. Como já citado anteriormente, todos os corpos-

Tabela I - Teor de alumina $\left(\mathrm{Al}_{2} \mathrm{O}_{3}\right)$, porosidade total (PT), porosidade aparente (PA) e a razão $\mathrm{R}\left(\mathrm{Al}_{2} \mathrm{O}_{3} / \mathrm{PT}\right)$ para os refratários estudados. [Table I - Alumina content $\left(\mathrm{Al}_{2} \mathrm{O}_{3}\right)$, total porosity $(\mathrm{PT})$, apparent porosity (PA) and ratio $R\left(\mathrm{Al}_{2} \mathrm{O}_{3} / \mathrm{PT}\right)$ for all refractories evaluated.]

\begin{tabular}{|c|c|c|c|c|}
\hline Nomenclatura & $\begin{array}{c}\mathrm{Al}_{2} \mathrm{O}_{3} \\
\text { (\%-peso) }\end{array}$ & $\begin{array}{l}\text { PT } \\
(\%)\end{array}$ & $\begin{array}{l}\text { PA } \\
(\%)\end{array}$ & $\begin{array}{l}\mathrm{R} \\
(-)\end{array}$ \\
\hline $\mathrm{I} 01$ & 35,81 & 71,14 & 60,78 & 0,50 \\
\hline $\mathrm{I} 02-1000^{\circ} \mathrm{C}^{*}$ & 36,24 & 78,36 & 72,45 & 0,46 \\
\hline $\mathrm{I} 03$ & 36,33 & 56,50 & 38,90 & 0,64 \\
\hline $\mathrm{I} 04$ & 40,10 & 57,88 & 55,66 & 0,69 \\
\hline $\mathrm{I} 05-1000^{\circ} \mathrm{C}$ & 41,85 & 46,66 & 44,62 & 0,90 \\
\hline D01 & 42,58 & 37,64 & 24,03 & 1,13 \\
\hline$\overline{\mathrm{D} 02}$ & 44,72 & 33,09 & 30,59 & 1,35 \\
\hline$\overline{\mathrm{D} 03}$ & 51,89 & 20,50 & 18,15 & 2,53 \\
\hline $\mathrm{I06}$ & 52,19 & 35,96 & 32,19 & 1,45 \\
\hline $\mathrm{C} 01$ & 52,86 & 20,28 & 16,21 & 2,61 \\
\hline D04 & 55,62 & 35,98 & 24,45 & 1,55 \\
\hline D05 & 60,86 & 22,45 & 20,19 & 2,71 \\
\hline D06 & 62,93 & 30,83 & 24,08 & 2,04 \\
\hline $\mathrm{C} 02$ & 64,54 & 24,62 & 18,82 & 2,62 \\
\hline D07-S & 67,11 & 21,90 & 20,07 & 3,06 \\
\hline D08 & 68,58 & 33,31 & 30,94 & 2,06 \\
\hline D07-P & 71,04 & 27,23 & 25,78 & 2,61 \\
\hline D09 & 71,06 & 29,38 & 25,92 & 2,42 \\
\hline D10 & 72,14 & 21,39 & 18,36 & 3,37 \\
\hline D11-P & 72,37 & 17,59 & 15,31 & 4,11 \\
\hline$\underline{\mathrm{D} 12}$ & 72,42 & 40,12 & 32,85 & 1,81 \\
\hline D11-S & 73,31 & 17,49 & 15,75 & 4,19 \\
\hline $\mathrm{D} 13$ & 75,93 & 23,51 & 21,37 & 3,23 \\
\hline $\mathrm{C} 03-1280^{\circ} \mathrm{C}$ & 76,44 & 19,60 & 14,82 & 3,90 \\
\hline$\overline{\mathrm{C} 04}$ & 82,68 & 22,33 & 18,74 & 3,70 \\
\hline D14-P & 84,31 & 22,19 & 20,23 & 3,80 \\
\hline D14-S & 84,41 & 19,60 & 17,92 & 4,31 \\
\hline$\overline{\mathrm{C} 05}$ & 89,59 & 17,50 & 15,44 & 5,12 \\
\hline $\mathrm{D} 15$ & 89,80 & 20,89 & 20,17 & 4,30 \\
\hline D16 & 90,34 & 33,74 & 33,24 & 2,68 \\
\hline $\mathrm{D} 17$ & 90,71 & 27,31 & 24,33 & 3,32 \\
\hline$\underline{\mathrm{D} 18}$ & 91,39 & 24,23 & 21,62 & 3,77 \\
\hline D19 & 92,33 & 18,11 & 14,79 & 5,10 \\
\hline D20 & 92,38 & 16,91 & 15,78 & 5,46 \\
\hline D21 & 93,48 & 15,25 & 12,16 & 6,13 \\
\hline
\end{tabular}

P: prensado S: socado * temperatura máxima de tratamento térmico 
de-prova foram tratados termicamente em temperaturas superiores àquelas empregadas na avaliação da condutividade térmica.

Tratamento dos Dados: A fim de avaliar as correlações existentes entre a condutividade térmica $(\mathrm{k})$ e o teor de alumina $\left(\mathrm{Al}_{2} \mathrm{O}_{3}\right)$ utilizou-se regressão linear. Um procedimento análogo foi empregado no ajuste da condutividade térmica em função da porosidade total (PT) e de k em função da porosidade aparente (PA).

A obtenção de uma equação que correlacionasse simultaneamente $\mathrm{k}, \mathrm{R}$ e $\mathrm{T}$ pode ser dividida em duas etapas. Na primeira, utilizou-se um procedimento numérico para a determinação da forma da equação de ajuste. Foram avaliados os parâmetros de ajuste através de linhas de tendência para $\mathrm{k}$ em função de $\mathrm{R}$ para cada uma das temperaturas de ensaio. Na segunda etapa, determinou-se o comportamento desses parâmetros em função da temperatura. Como o modelo obtido foi linear, utilizou-se um procedimento analítico para confirmar os resultados obtidos pelo método numérico sem a interferência de convergência.

A qualidade do ajuste foi avaliada através de quadros de análise de variância (ANOVA), como no modelo apresentado na Tabela II, e do coeficiente de correlação linear ao quadrado $\left(\mathrm{r}^{2}\right)$ cujas definições são apresentados a seguir.

Tabela II - Quadro de análise de variância.

[Table II - Variance analysis.]

Fonte de Variação graus de liberdade soma dos quadrados

\begin{tabular}{lll}
\hline $\begin{array}{l}\text { Regressão } \\
\text { (modelo ajustado) }\end{array}$ & $\mathrm{p}-1$ & $\mathrm{SQR}$ \\
\hline Resíduos & $\mathrm{n}-\mathrm{p}$ & $\mathrm{SQE}$ \\
\hline Total & $\mathrm{n}-1$ & $\mathrm{SQT}$ \\
\hline
\end{tabular}

Nas quais:

$\mathrm{SQR}=\sum_{\mathrm{i}=1}^{\mathrm{n}}\left(\hat{\mathrm{y}}_{\mathrm{i}}-\overline{\mathrm{y}}\right)^{2}:$ soma dos quadrados devida à regressão; $\mathrm{SQE}=\sum_{\mathrm{i}=1}^{\mathrm{n}}\left(\mathrm{y}_{\mathrm{i}}-\hat{\mathrm{y}}\right)^{2}:$ soma dos quadrados dos resíduos; $\mathrm{SQT}=\sum_{\mathrm{i}=1}^{\mathrm{n}}\left(\mathrm{y}_{\mathrm{i}}-\overline{\mathrm{y}}\right)^{2}:$ soma dos quadrados total; $\mathrm{y}_{\mathrm{i}}$ : i-ésimo valor avaliado experimentalmente; $\hat{\mathrm{y}}_{\mathrm{i}}$ : i-ésimo valor predito pelo modelo; $\overline{\mathrm{y}}=\sum_{\mathrm{i}=1}^{\mathrm{n}} \frac{\mathrm{y}_{\mathrm{i}}}{\mathrm{n}}:$ média dos dados observados e n: número total de dados experimentais.

A proporção da variância dos valores em torno da média explicada pelo modelo ajustado pode ser expressa pelo coeficiente de correlação linear ao quadrado $\left(\mathrm{r}^{2}\right)$ através da equação $(\mathrm{C})$ :

$$
\mathrm{r}^{2}=\frac{\mathrm{SQR}}{\mathrm{SQT}}
$$

\section{RESULTADOS E DISCUSSÃO}

A partir dos teores de alumina e das características físicas apresentadas na Tabela I buscaram-se correlações entre a condutividade térmica, o teor de alumina, a porosidade e a temperatura.

Correlação com o Teor de Alumina. Todos os ajustes de parâmetros das equações obtidas neste trabalho foram realizados através do método dos mínimos quadrados. Nesta etapa, foram avaliados refratários com teor de alumina entre 35,81\%-p e 93,48\%-p;
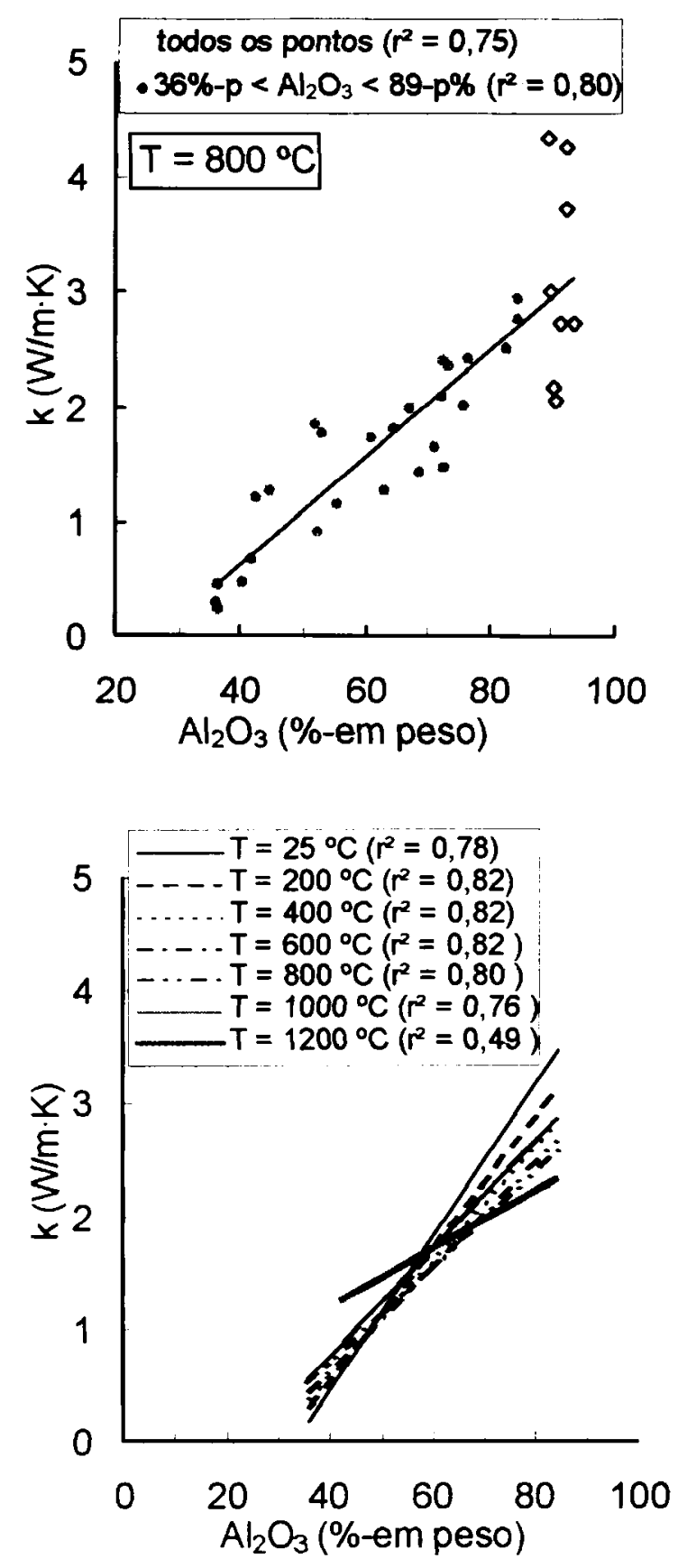

Figura 1: Condutividade térmica (k) em função do teor de alumina: (a) $\mathrm{T}=800^{\circ} \mathrm{C}$ e (b) linhas de tendência para a condutividade térmica em função do teor de alumina para as demais temperaturas de ensaio e 36\%-p $<\mathrm{Al}_{2} \mathrm{O}_{3}<89 \%$-p.

[Figure 1: Thermal conductivity ( $k$ ) as function of alumina content: (a) $T=800{ }^{\circ} \mathrm{C}$ and (b) trend lines of thermal conductivity as function of alumina content to all measured temperatures and $36 \mathrm{wt} \% \leq \mathrm{Al}_{2} \mathrm{O}_{3} \leq 89 \mathrm{wt} \%$.] 
totalizando 209 pontos experimentais. As curvas para a temperatura de $1200{ }^{\circ} \mathrm{C}$ apresentam um número menor de pontos, uma vez que os materiais caracterizados como isolantes (nomenclatura I na Tabela I), foram tratados termicamente em temperaturas inferiores a $1200^{\circ} \mathrm{C}$.

A Fig. 1a apresenta a condutividade térmica $(\mathrm{k})$ em função do teor de alumina $\left(\mathrm{Al}_{2} \mathrm{O}_{3}\right.$, em porcentagem em peso) para a temperatura de $800{ }^{\circ} \mathrm{C}$ enquanto a Fig. $1 \mathrm{~b}$ apresenta as linhas de tendência para todas temperaturas de tratamento térmico.

A Fig. 1a mostra que, embora exista uma correlação linear entre a condutividade térmica e o teor de alumina, os pontos experimentais se encontram dispersos em torno da curva ajustada, principalmente para teores de alumina superiores a $89 \%$-p. Mesmo desconsiderando-se os materiais com teor de alumina superior a $89 \%$-p nota-se pela Fig. 1b que o valor do coeficiente de correlação linear ao quadrado foi inferior a 0,90 para todas as temperaturas de ensaio. Essa dispersão também foi encontrada por Chien e colaboradores [6] para refratários comerciais sílico-aluminosos e aluminosos com teor de alumina entre 44\%-p e 93\%-p.

Segundo Chien e colaboradores [6], uma possível explicação para estes baixos valores de $r^{2}$ pode ser a utilização do teor de alumina obtido através da análise química dos refratários sem se considerar as fases cristalinas presentes. Outra possibilidade é a necessidade de se considerar, além do teor de alumina, a influência da porosidade sobre a condutividade térmica.

Correlação com a Porosidade. Utilizando-se os valores de porosidade total (PT) e porosidade aparente (PA) apresentados na Tabela I, buscaram-se correlações entre a condutividade térmica e a porosidade. A condutividade térmica exibiu um comportamento aproximadamente exponencial tanto com a porosidade total quanto com a porosidade aparente como apresentado nas Figs. 2 e 3.

De modo semelhante ao teor de alumina, verifica-se pelas Figs. $2 \mathrm{a}$ e $3 \mathrm{a}$ que os maiores espalhamentos ocorreram para teores de alumina superiores a $89 \%$-p. Nota-se que desconsiderando-se os materiais com $\mathrm{Al}_{2} \mathrm{O}_{3}>89 \%$-p houve um aumento nos valores de $\mathrm{r}^{2}$. Chan e colaboradores [7] também não encontraram correlação entre a condutividade térmica e a porosidade aparente para refratários monolíticos com 90\%-p $<\mathrm{Al}_{2} \mathrm{O}_{3}<96 \%$-p e $19,38 \%<\mathrm{PA}<25,95 \%$.

Considerando que, neste trabalho, foram utilizadas faixas de porosidade $(17,50 \%<\mathrm{PT}<78,36 \%)$ e teor de alumina $(35,81 \%$ $\left.\mathrm{p}<\mathrm{Al}_{2} \mathrm{O}_{3}<93,48 \%-\mathrm{p}\right)$ mais abrangentes, optou-se por desconsiderar os materiais com teor de alumina acima de $89 \%$ para não sacrificar a qualidade dos ajustes. Doravante serão analisados somente os materiais com teor de alumina menor que $89 \%$-p, reduzindo o número de pontos experimentais para 181.

As Figs. $2 b$ e $3 b$ apresentam as linhas de tendência e os valores do coeficiente de correlação linear ao quadrado para o ajuste de $\mathrm{k}$ em função de PT e k em função de PA, respectivamente, em cada uma das temperaturas de ensaio. Comparando as Fig. 2 e Fig. 3, observa-se que o coeficiente de correlação linear ao quadrado para $\mathrm{k}$ em função de PT foi maior que o apresentado pela correlação entre a k em função de PA para todas as temperaturas de ensaio. Esse resultado já era esperado, uma vez que PT considera tanto o volume dos poros abertos quanto dos poros fechados enquanto PA considera somente aquele dos poros abertos.

Correlação com entre a condutividade térmica e a razão R. É importante lembrar que a condutividade térmica é função tanto da composição química quanto da porosidade do material. Deste modo,


Figura 2: Condutividade térmica (k) em função da porosidade total (PT): (a) para $\mathrm{T}=800{ }^{\circ} \mathrm{C}$ e (b) para as demais temperaturas de ensaio e $36 \%$ - $\mathrm{p} \leq \mathrm{Al}_{2} \mathrm{O}_{3} \leq 89 \%$-p.

[Figure 2: Thermal conductivity ( $k$ ) as function of total porosity (PT): (a) $T=800{ }^{\circ} \mathrm{C}$ and $(\mathrm{b})$ trend lines to all measured temperatures and $36 \mathrm{wt} \% \leq \mathrm{Al}_{2} \mathrm{O}_{3} \leq 89 \mathrm{wt} \%$.]

buscou-se um parâmetro que correlacionasse simultaneamente ambas as variáveis. Para tanto, utilizou-se a razão R, dada por:

$$
\mathrm{R}=\frac{\text { Teor de alumina }}{\text { Porosidade total }}=\frac{\mathrm{Al}_{2} \mathrm{O}_{3}}{\mathrm{PT}}
$$

A Fig. 4a apresenta a condutividade térmica em função da razão $\mathrm{R}$ para a temperatura $\mathrm{T}=800{ }^{\circ} \mathrm{C}$ e a Fig. $4 \mathrm{~b}$ apresenta as linhas de tendência para o ajuste de $\mathrm{k}$ em função de $\mathrm{R}$ em cada uma das temperaturas de ensaio.

A qualidade dos ajustes pode ser verificada através da Fig. 4b onde os valores do coeficiente de correlação linear ao quadrado $\left(\mathrm{r}^{2}\right)$ foram sempre superiores a 0,89 .

Uma das principais vantagens advindas da utilização da razão $\mathrm{R}$ é correlacionar a condutividade térmica através de uma reta como 

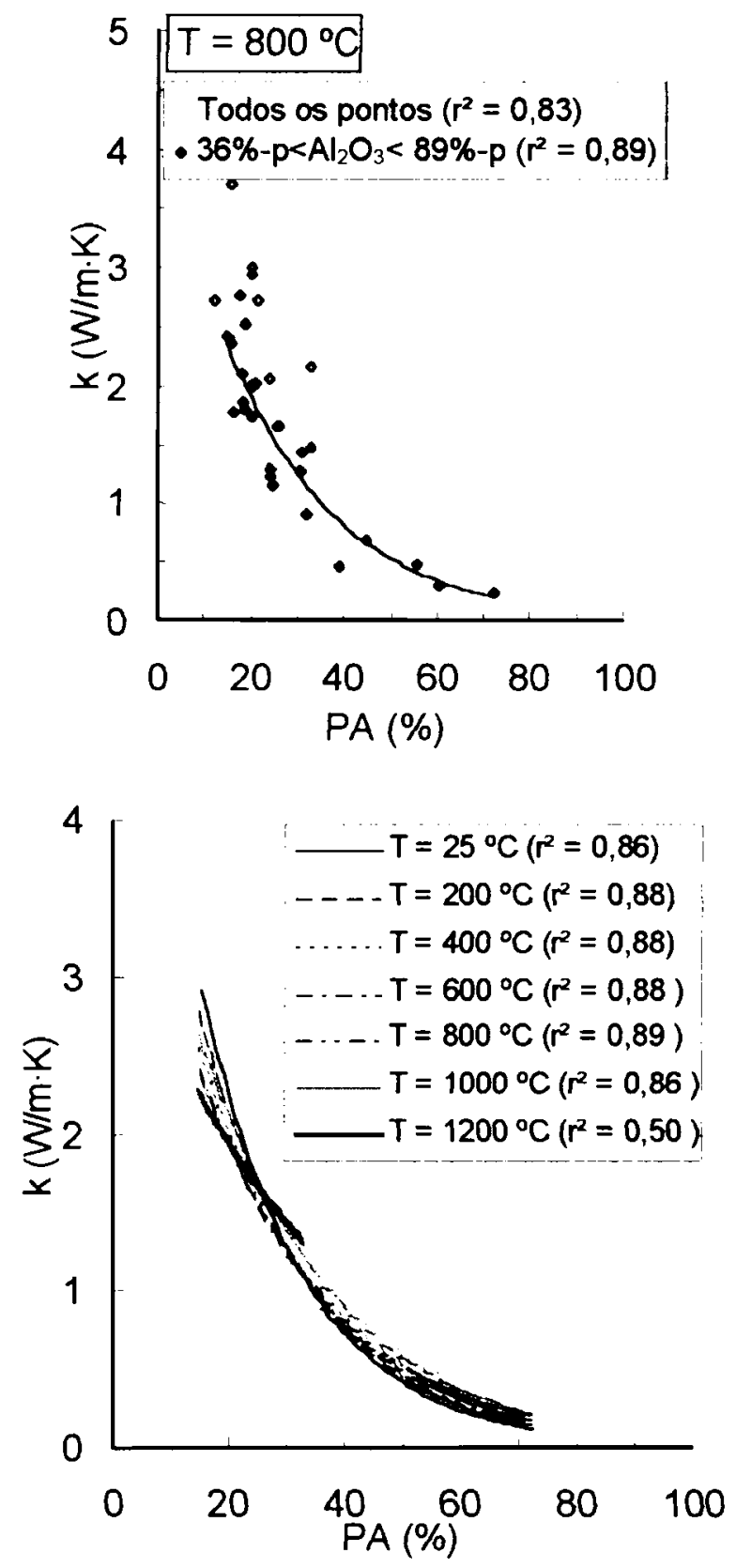

Figura 3: Condutividade térmica em função da porosidade aparente (PA): (a) para $\mathrm{T}=800{ }^{\circ} \mathrm{C}$ e (b) para todas as temperaturas de ensaio e $36 \%$ $\mathrm{p} \leq \mathrm{Al}_{2} \mathrm{O}_{3} \leq 89 \%$-p.

[Figure 3: Thermal conductivity ( $k$ ) as function of apparent porosity (PA): (a) $T=800{ }^{\circ} \mathrm{C}$ and (b) trend lines to all measured temperatures and $36 \mathrm{wt} \% \leq \mathrm{Al}_{2} \mathrm{O}_{3} \leq 89 \mathrm{wt} \%$.]

a equação (E), ao invés de correlações exponenciais como nos casos das correlações de k em função de PT.

$$
\mathrm{k}=\mathrm{q}_{1}+\mathrm{q}_{2} \cdot \mathrm{R}
$$

Conforme descrito anteriormente, o procedimento numérico foi utilizado para obter a forma das correlações desejadas, tendo-se concluído que a equação (E) é a forma mais simples e representativa para a correlação entre a condutividade térmica e a razão $\mathrm{R}$ para cada temperatura.

A Tabela III apresenta o número de pontos experimentais, os valores dos parâmetros ajustados $\left(\mathrm{q}_{1}\right.$ e $\left.\mathrm{q}_{2}\right)$ e do coeficiente de
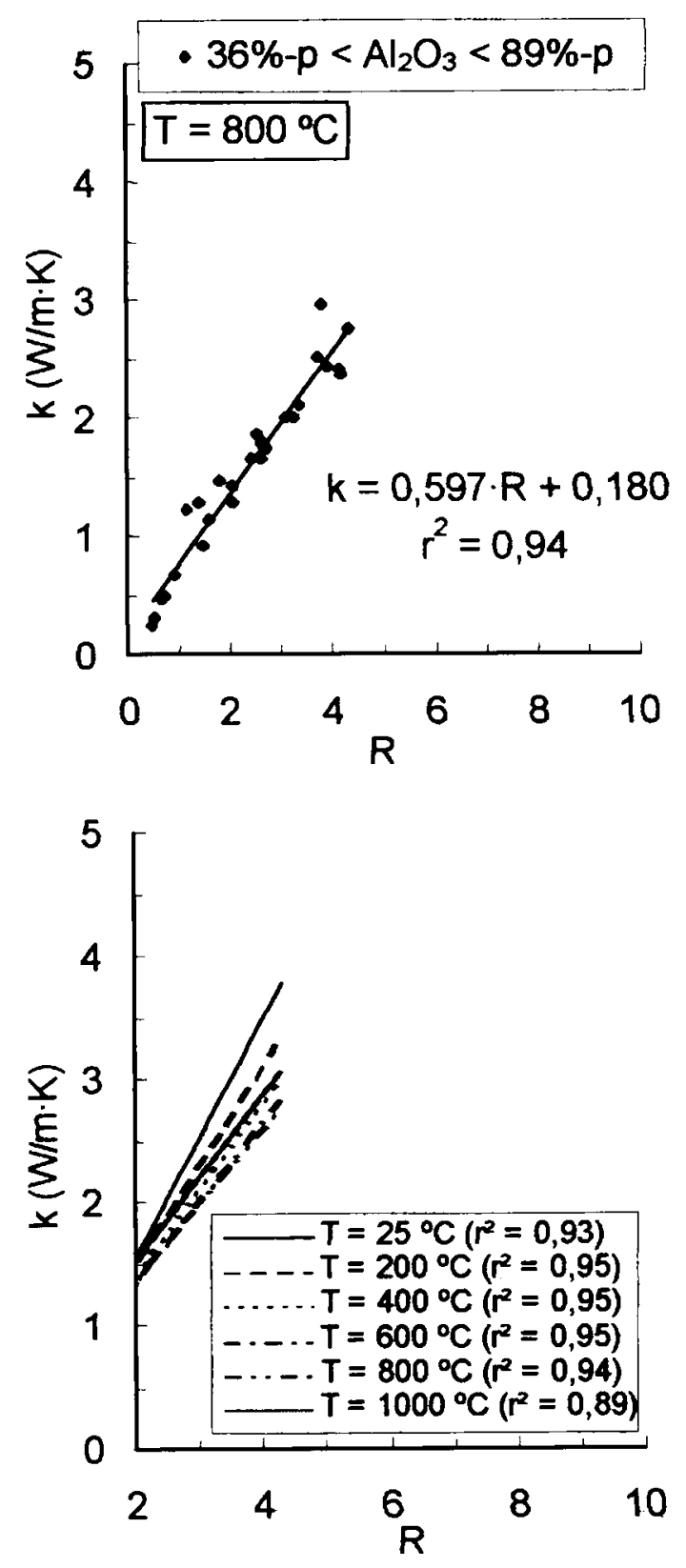

Figura 4: Condutividade térmica em função da razão $\mathrm{R}$ (36\%$\mathrm{p} \leq \mathrm{Al}_{2} \mathrm{O}_{3} \leq 89 \%$-p; $16 \% \leq \mathrm{PT} \leq 72 \%$ ): (a) para $\mathrm{T}=800{ }^{\circ} \mathrm{C}$ e (b) para todas as temperaturas de ensaio e $36 \%$-p $\leq \mathrm{Al}_{2} \mathrm{O}_{3} \leq 89 \%$-p.

[Figure 4: Thermal conductivity (k) as function ratio $R$ (36 wt \% $\leq \mathrm{Al}_{2} \mathrm{O}_{3} \leq 89 \mathrm{wt} \% ; 16 \% \leq P T \leq 72 \%$ ): (a) $\mathrm{T}=800{ }^{\circ} \mathrm{C}$ and $(b)$ trend lines to all measured temperatures and $36 \mathrm{wt} \% \leq \mathrm{Al}_{2} \mathrm{O}_{3} \leq 89 \mathrm{wt} \%$.]

correlação linear ao quadrado para o ajuste da condutividade térmica em função da razão R para cada uma das temperaturas de ensaio.

Na segunda etapa, utilizaram-se os dados da Tabela III para ajustar os parâmetros $\mathrm{q}_{1}$ e $\mathrm{q}_{2}$ em função da temperatura. As equações de ajuste obtidas são apresentadas abaixo:

$\mathrm{q}_{1}=-0,370+1,25 \cdot 10^{-3} \cdot \mathrm{T}-6,82 \cdot 10^{-7} \cdot \mathrm{T}^{2} ; \mathrm{r}^{2}=0,98$

$\mathrm{q}_{2}=0,984-1,03 \cdot 10^{-3} \cdot \mathrm{T}+7,03 \cdot 10^{-7} \cdot \mathrm{T}^{2} ; \mathrm{r}^{2}=1,00$ 
Tabela III - Número de pontos experimentais por temperatura, parâmetros $\mathrm{q}_{1}, \mathrm{q}_{2}$ e o coeficiente de correlação ao quadrado $\left(\mathrm{r}^{2}\right)$ obtidos pelo ajuste numérico em duas etapas para os refratários avaliados.

[Table III - Number of experimental data, parameters $q_{1}$ and $q_{2}$ and square linear correlation coefficient $\left(r^{2}\right)$ obtained by the twosteps numerical procedure for the evaluated refractories.]

\begin{tabular}{lcccc}
\hline $\mathrm{T}\left({ }^{\circ} \mathrm{C}\right)$ & Número & $\mathrm{q}_{1}$ & $\mathrm{q}_{2}$ & $\mathrm{r}^{2}$ \\
& de pontos & & & \\
\hline 25 & 26 & $-0,3656$ & 0,9644 & 0,9346 \\
\hline 200 & 27 & $-0,1046$ & 0,7918 & 0,9456 \\
\hline 600 & 27 & 0,0305 & 0,6858 & 0,9466 \\
\hline 800 & 27 & 0,1110 & 0,6264 & 0,9473 \\
\hline 1000 & 27 & 0,1804 & 0,5970 & 0,9386 \\
\hline 1200 & 27 & 0,2142 & 0,6537 & 0,8850 \\
\hline
\end{tabular}

Numero total de pontos $=181$

Como já citado, devido ao menor número de pontos experimentais, as curvas para a temperatura de $1200{ }^{\circ} \mathrm{C}$ apresentaram comportamento distinto das demais temperaturas de ensaio, sendo por isso, excluídas dos ajustes dos parâmetros $\mathrm{q}_{1}$ e $\mathrm{q}_{2}$ em função da temperatura.

A equação final obtida pelo método numérico para a correlação entre a condutividade térmica, a razão $\mathrm{R}$ e a temperatura pode ser obtida substituindo-se $\mathrm{q}_{1}(\mathrm{~T})$ e $\mathrm{q}_{2}(\mathrm{~T})$ na equação $(\mathrm{E})$, resultando em:

$$
\begin{gathered}
\mathrm{k}=-0,3696+0,001251 \cdot \mathrm{T}-6,8237 \cdot 10^{-7} \cdot \mathrm{T}^{2}+ \\
+\left(0,9837-0,001035 \cdot \mathrm{T}+7,0289 \cdot 10^{-7} \cdot \mathrm{T}^{2}\right) \cdot \mathrm{R}
\end{gathered}
$$

$$
\begin{gathered}
{\left[25^{\circ} \mathrm{C} \leq \mathrm{T} \leq 1000{ }^{\circ} \mathrm{C}, 35,81 \%-\mathrm{p} \leq \mathrm{Al}_{2} \mathrm{O}_{3} \leq 89 \%-\mathrm{p} ;\right.} \\
17,49 \% \leq \mathrm{PT} \leq 78,36 \% \text { para k em } \mathrm{W} / \mathrm{m} \cdot \mathrm{K}]
\end{gathered}
$$

O ajuste dos parâmetros da equação acima pelo método dos mínimos quadrados também foi realizado analiticamente, uma vez que o procedimento numérico fornece apenas uma das possíveis soluções para os parâmetros da função de ajuste, enquanto que se utilizando procedimento analítico a solução é absoluta. Como no procedimento analítico foram avaliadas matrizes de grandes dimensões, utilizou-se o programa Mapple V (Release 4.0 - Waterloo Mapple Inc.) para a obtenção dos parâmetros da função de ajuste.

A Tabela IV apresenta os valores dos parâmetros e a tabela de análise de variância (neste caso, são apresentadas somente as somas dos quadrados) obtidos através do métodos dos mínimos quadrados pelo processo numérico e pelo processo analítico.

Comparando-se os parâmetros obtidos pelo método dos mínimos quadrados através dos procedimentos numérico e analítico, nota-se que os valores são muito próximos, diferindo somente após a terceira casa decimal, indicando que além de ser útil na determi-
Tabela IV - Coeficientes estimados para a equação $(\mathrm{H})$. [Table IV - Estimated parameters for equation (H).]

\begin{tabular}{lcc}
\hline Parâmetro & Método Numérico & Método Analítico \\
\hline$\gamma_{1}$ & $-0,3696$ & $-0,3697$ \\
$\gamma_{2}$ & 0,001251 & 0,001252 \\
\hline$\gamma_{3}$ & $-6,8237 \cdot 10^{-7}$ & $-6,8274 \cdot 10^{-7}$ \\
\hline$\gamma_{4}$ & 0,9837 & 0,9838 \\
\hline$\gamma_{5}$ & 0,001035 & $-0,00103516$ \\
\hline$\gamma_{6}$ & $7,0289 \cdot 10^{-7}$ & $7,0318 \cdot 10^{-7}$ \\
\hline SQT & Análise de Variância & 131,3263 \\
\hline SQR & 131,3263 & 122,6114 \\
\hline SQE & 122,4307 & 8,7148 \\
\hline$r^{2}$ & 8,7149 & 0,934 \\
\hline & 0,932 & \\
\hline
\end{tabular}

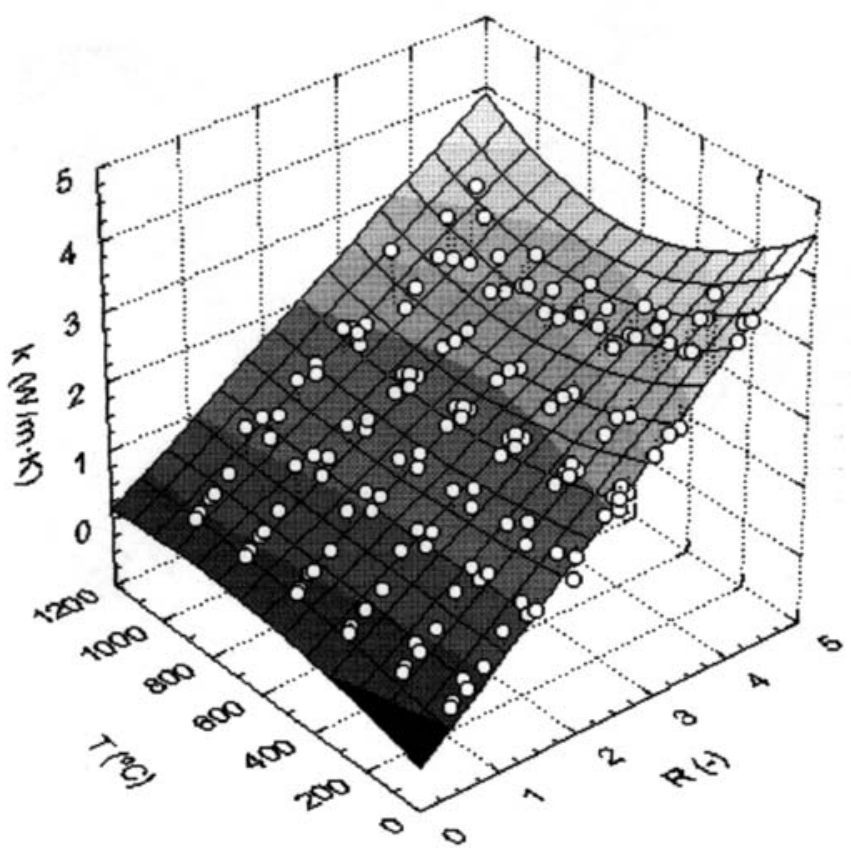

Figura 5: Representação tridimensional da correlação entre a condutividade térmica, a razão $\mathrm{R}$ e a temperatura.

[Figure 5: Three dimensional plot for the correlation between the thermal conductivity ( $k$ ) as function ratio $R$ and temperature.]

nação da forma da função de ajuste, o método numérico pôde (neste caso) ser utilizado para determinar os parâmetros da função de ajuste. É importante lembrar que quando são utilizadas funções mais complexas, o procedimento numérico e o analítico podem levar a valores bastante distintos, sendo que os últimos são mais estáveis. 
Assim, recomenda-se, sempre que possível, a utilização do método analítico na avaliação do método dos mínimos quadrados.

Na Fig. 5 tem-se uma representação tridimensional da correlação entre a condutividade térmica, a razão $\mathrm{R}$ e a temperatura. A curva de ajuste foi obtida a partir dos dados da Tabela IV para o método analítico.

$\mathrm{O}$ ajuste foi realizado para temperaturas entre $25^{\circ} \mathrm{C}$ e $1000{ }^{\circ} \mathrm{C}$ e o coeficiente de correlação linear ao quadrado $\left(r^{2}\right)$ igual a 0,93 . Não foi possível incluir os pontos para a temperatura de $1200^{\circ} \mathrm{C}$, uma vez que a inclusão destes pontos experimentais acarretava grandes desvios em relação a curva de ajuste.

\section{CONCLUSÕES}

Foram avaliadas as correlações entre a condutividade térmica, o teor de alumina $\left(\mathrm{Al}_{2} \mathrm{O}_{3}\right)$ e a porosidade (PA e PT) em refratários sílico-aluminosos e aluminosos com 35,81\%-p $\leq \mathrm{Al}_{2} \mathrm{O}_{3} \leq 93,48 \%$-p e porosidade total entre $15 \%$ e $81 \%$. Para avaliar simultaneamente a influência do teor de alumina e da porosidade total sobre a condutividade térmica foi utilizada a razão $\mathrm{R}=\frac{\text { Teor de alumina }}{\text { Porosidade total }}$. Escolheu-se a razão $\mathrm{R}$ porque o ajuste da condutividade térmica em função de $\mathrm{R}$ foi linear e apresentou valores de coeficiente de correlação linear ao quadrado $\left(\mathrm{r}^{2}\right)$ superiores aos encontrados para os ajustes individuais entre a condutividade térmica e a porosidade (PT e PA). Embora o ajuste da condutividade térmica em função do teor de alumina também tenha sido linear, observou-se uma grande dispersão nos dados experimentais. Por outro lado, os ajustes de $\mathrm{k}$ em função de PT e k em função de PA apresentaram coeficiente de correlação linear ao quadrado $\left(\mathrm{r}^{2}\right)$ superior a 0,90 , mas eram desvantajosos por serem relações exponenciais.

Os resultados permitiram ajustar uma equação que correlacionou a condutividade térmica simultaneamente como função da razão $\mathrm{R}$ e da temperatura . Foram comparados os procedimentos numérico e o analítico para a obtenção dos parâmetros da função de ajuste. Embora, neste caso ambos os procedimentos tenham levado a parâmetros próximos, foi lembrado que o procedimento analítico fornece valores mais estáveis.

O coeficiente de correlação linear ao quadrado avaliado para a equação obtida com o ajuste dos 161 pontos experimentais utilizados foi de $r^{2}=0,93$, e o erro médio percentual foi de $14 \%$, indicando a boa qualidade do ajuste. As principais vantagens desta equação foram abordar simultaneamente a influência do teor de alumina
$\left(\mathrm{Al}_{2} \mathrm{O}_{3}\right)$ e da porosidade total $(\mathrm{PT})$ sobre a condutividade térmica $(\mathrm{k})$, além de permitir generalizar a correlação entre k e a razão $R$ para uma maior faixa de temperaturas e não somente para temperaturas específicas.

A boa qualidade do ajuste obtido através do modelo empírico desenvolvido neste trabalho permite concluir que a utilização da razão $\mathrm{R}$ que considera os efeitos tanto do teor de alumina quanto da porosidade total pode ser mais significativa para a condutividade térmica de cerâmicas refratárias do que a utilização do teor de alumina ou quantidade de coríndon isoladamente.

\section{AGRADECIMENTOS}

Os autores agradecem à FAPESP, à CAPES e à Cerâmica SAFFRAN S.A. pelo apoio neste trabalho

\section{REFERÊNCIAS}

[1] K. W. Cowling, A. Elliott, W. T. Hale. "Note on the relationship between bulk density and thermal conductivity in refractory insulating bricks". Transactions of British Ceramic Society, 53 (1954) 461-73.

[2] R. W. Wallace, G. H. Criss, "Thermal conductivity of castable refractories in relation to bulk density". Am. Ceram. Soc. Bull. 47, 2 (1968) 176-9.

[3] G. Routschka et al., "Thermal conductivity of refractory castables". Interceram 37, 3 (1988) 24-33.

[4] M. M. Akiyoshi, M. G. da Silva, M. D. M. Innocentini, C. Pagliosa Neto, V. C. Pandolfelli, "Variáveis envolvidas na avaliação da condutividade térmica de materiais refratários através da técnica de fio quente paralelo". Anais do $44^{\circ}$ Congresso Brasileiro de Cerâmica, S. Pedro, S.P., I CD-ROM (2000).

[5] M. M. Akiyoshi, M. G. da Silva, A. P. da Silva, V. C. Pandolfelli, " Condutividade térmica e sua correlação com a temperatura e a massa específica volumétrica de materiais refratários sílicoaluminosos e aluminosos". Cerâmica 47, 301 (2001) 19-17

[6] Y-T Chien, T-F Lee, Y-C Ko, "Thermal conductivity of fireclay and high-alumina refractory brick". Am. Ceram. Soc. Bull. 61, 7 (1982) 718-24.

[7] C-F Chan, Y-C Ko, "Influence of coarse aggregate content on thermal conductivity of alumina-spinel castables". J. Am. Ceram. Soc. 79, 11 (1996) 2961-4.

(Rec.16/02/01, Ac. 22/06/01) 